\title{
The role of small RNAs in wide hybridisation and allopolyploidisation between Brassica rapa and Brassica nigra
}

Muhammad Awais Ghani ${ }^{1,2}$, Junxing Li ${ }^{1,2}$, Linli Rao ${ }^{1,2}$, Muhammad Ammar Raza ${ }^{1,2}$, Liwen Cao ${ }^{1,2}$, Ningning Yu ${ }^{1,2}$, Xiaoxia Zou ${ }^{1,2}$ and Liping Chen ${ }^{1,2^{*}}$

\begin{abstract}
Background: An allopolyploid formation consists of the two processes of hybridisation and chromosome doubling. Hybridisation makes a different genome combined in the same cell, and genome "shock" and instability occur during this process, whereas chromosome doubling results in doubling and reconstructing the genome dosage. Recent studies have demonstrated that small RNAs, play an important role in maintaining the genome reconstruction and stability. However, to date, little is known regarding the role of small RNAs during the process of wide hybridisation and chromosome doubling, which is essential to elucidate the mechanism of polyploidisation. Therefore, the genetic and DNA methylation alterations and changes in the siRNA and miRNA were assessed during the formation of an allodiploid and its allotetraploid between Brassica rapa and Brassica nigra in the present study.
\end{abstract}

Results: The phenotypic analysis exhibited that the allotetraploid had high heterosis compared with their parents and the allodiploid. The methylation-sensitive amplification polymorphism (MSAP) analysis indicated that the proportion of changes in the methylation pattern of the allodiploid was significantly higher than that found in the allotetraploid, while the DNA methylation ratio was higher in the parents than the allodiploid and allotetraploid. The small RNAs results showed that the expression levels of miRNAs increased in the allodiploid and allotetraploid compared with the parents, and the expression levels of siRNAs increased and decreased compared with the parents B. rapa and B. nigra, respectively. Moreover, the percentages of miRNAs increased with an increase in the polyploidy levels, but the percentages of siRNAs and DNA methylation alterations decreased with an increase in the polyploidy levels. Furthermore, qRT-PCR analysis showed that the expression levels of the target genes were negatively corrected with the expressed miRNAs.

Conclusions: The study showed that siRNAs and DNA methylation play an important role in maintaining the genome stability in the formation of an allotetraploid. The miRNAs regulate gene expression and induce the phenotype variation, which may play an important role in the occurrence of heterosis in the allotetraploid. The findings of this study may provide new information for elucidating that the allotetraploids have a growth advantage over the parents and the allodiploids.

Keywords: Wide hybridisation, Allopolyploidisation, DNA methylation, Small RNAs

\footnotetext{
* Correspondence: chenliping@zju.edu.cn

${ }^{1}$ Department of Horticulture, College of Agriculture and Biotechnology,

Zhejiang University, Yuhangtang Road No.866, Hangzhou 310058, Zhejiang

Province, P. R. China

${ }^{2}$ Key Laboratory of Horticultural Plant Growth, Development, and

Biotechnology, Agricultural Ministry of China, Hangzhou 310058, P. R. China
} 


\section{Background}

Wide hybridisation and polyploidisation is a common phenomenon in plant evolution that results in the formation of new species [1-4]. Wide hybrids often exhibit more vigorous growth than their parents, and this effect is mainly demonstrated by increases in drought tolerance, pest resistance, flowering time, organ size and biomass, among other factors [5-7]. Furthermore, polyploids show novel traits that are not present in their diploid progenitors [8]. For example, allotetraploid cotton (genomes: AADD) produces more abundant and higher-quality fibres, and this effect is derived from their AA and DD extant diploid species [9]. However, wide hybrids also exhibit the disadvantage of infertility, as was observed with the wide hybridisation combination of B. rapa and B. oleracea [10]. However, despite this finding, the growth and adaptability advantages of polyploidy have always been a puzzling phenomenon, and the underlying molecular mechanisms are among the most interesting subjects in plant breeding.

Allopolyploid formation consists of two processes, namely, hybridisation and chromosome doubling. Hybridisation involves a different genomic combination in the same cells and genomes experience "genomic shock", whereas chromosome doubling doubles and restructures the genome dosage $[11,12]$. Recent studies have shown that small RNAs, particularly the 24-nt siRNAs, play an important role in genome reconstruction and stabilisation [13]. The role of 24-nt siRNAs is primarily reflected in two aspects. The first aspect is the modification of transposons and repetitive sequences for the maintenance of genome stability, which is mediated by RNA-dependent DNA methylation, and the other aspect is the cis-regulation of gene expression via the transposon gene fragment in the gene near the region (such as the promoter expression area) [14-18]. The reduction of siRNAs in $F_{1}$ may induce phenotypic and genomic instability in Arabidopsis allotetraploids [13,19]. In interspecific hybrids of Arabidopsis, the siRNA populations underwent rapid changes in the $F_{1}$ allotetraploid, but stability was maintained in the $F_{7}$ allotetraploid, in which the DNA and chromatin were significantly modified [13]. However, in wheat, the number of 24-nt siRNA transcripts significantly decreased in the hexaploid compared with that obtained in the parents, and this decrease was accompanied by a decrease in the DNA chain CpG island methylation levels [20]. Thus, these studies concluded that a decrease in the DNA methylation levels may be one of the causes of genomic instability in allopolyploids during the early stage [21,13]. This phenomenon of changes to the siRNAs with an increase in the polyploidy level suggests that siRNAs may play a key role in genomic reconstruction and stability after chromosome doubling. However, little is known regarding how siRNAs play this role during wide hybridisation (with different genome combined) and chromosome doubling (the doubling of the genome dosage).

miRNAs are the primary mediators of the transregulation of gene expression [22]. Gene silencing mediated by miRNAs is an important strategy used at the post-transcriptional level of gene regulation [23,24]. Furthermore, miRNAs are conserved in evolution but become active in polyploidisation [25,13]. Changes in the miRNA expression levels can affect the expression of the target genes, and this effect is considered to be one of the main causes that results in the phenotypic variation of the polyploidy [21,26,27,13]. In Arabidopsis, the number of miRNAs in allotetraploids is higher than those observed in their autotetraploid parents [13]. A similar phenomenon was also found in the synthesis of hexaploid wheat [20]: the number of miRNAs increased with an increase in the ploidy level, and this effect was not associated with the gene dosage balance hypothesis. This phenomenon showed that other mechanisms, such as cis-epigenetic regulation, may exist. Thus, small RNAs, which are a product of non-coding RNAs, are involved in regulating gene expression and have become an important factor of gene expression during allopolyploidisation. However, little is known regarding the changes in miRNAs and their regulation of gene expression and phenotypic variation during wide hybridisation and chromosome doubling, and these data are essential for elucidating the mechanism of heterosis.

In a previous study, we performed wide hybridisation between $B$. rapa (genome: $\mathrm{AA}, 2 \mathrm{n}=20$ ) and $B$. nigra (genome: $\mathrm{BB}, 2 \mathrm{n}=16$ ) and obtained an allodiploid (genome: $\mathrm{AB}$ ) and allotetraploid (genome: $\mathrm{AABB}$ ). In addition, we showed that chromosome doubling resulted in higher levels of genetic and phenotypic variation compared with wide hybridisation [28]. In this study, we first analysed the allodiploids and allotetraploids using sequence-related amplified polymorphism (SRAP) and methylation-sensitive amplification polymorphism (MSAP) to determine the differences in the genetic changes and epigenetic alterations between wide hybridisation and chromosome doubling. Second, the allodiploids and allotetraploids were analysed through the high-throughput sequencing of small RNAs to determine how the changes in small RNAs occur during these two processes. Different genomes were combined, the genome dosage was doubled, and the correlation between the siRNA and DNA methylation at different polyploid levels was assessed. Third, the different expression levels of known miRNAs and their target genes were analysed to explore how miRNAs and their target genes affect the different phenotypes of the allodiploids and allotetraploids. 


\section{Results}

Phenotypic analysis of the parents and their wide hybrids In our previous study, the wide hybridisation of B. rapa (genome $\mathrm{AB}$ ) and $B$. nigra (genome $\mathrm{AA}$ ) was performed, and the allodiploid (genome $\mathrm{AB}$ ) and allotetraploid (genome $\mathrm{AABB}$ ) were obtained. We aimed to determine the phenotypic differences between the wide hybrid and their parents. The characteristics of the allodiploid and allotetraploid and their parents were compared (Figures 1 and 2). The results showed that allotetraploids had a high leaf length and flower size compared with their parents and the allodiploids (Figures 1 and 2). In our previous study, we found that allotetraploids had greater vigour than their parents and allodiploids [28]. Thus, allotetraploids had high heterosis compared with their parents and the allodiploids.

\section{DNA methylation patterns of the parents and their wide hybrids}

To elucidate the epigenetic mechanisms related to the processes of hybridisation and polyploidisation, methylationsensitive amplification polymorphism (MSAP) analysis was used to analyse the parents and their allodiploid $(\mathrm{AB})$ and allotetraploid (AABB). After treatment with doublerestriction EcoRI/MspI or EcoRI /HpaII, the amplified fragments were classified as one of four types: (a) nonmethylated in all of the samples, (b) methylated in all of the samples, (c) demethylated in the hybrids compared with the parents, and (d) hyper-methylated in the hybrids compared with the parents (Additional file 1).

In this study, 1449 reproducible and clear loci were obtained using 36 primer pairs (Additional file 2). These 1449 loci were classified into four major groups (a-d),
Brassica rapa (genome: AA)

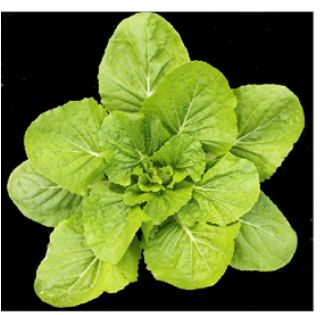

Brassica nigra (genome: $\mathrm{BB}$ )

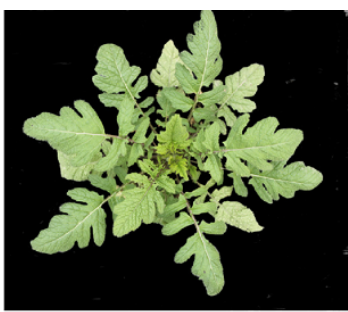

Allodiploid $\mathrm{F}_{1}$ (genome: $\mathrm{AB}$ )
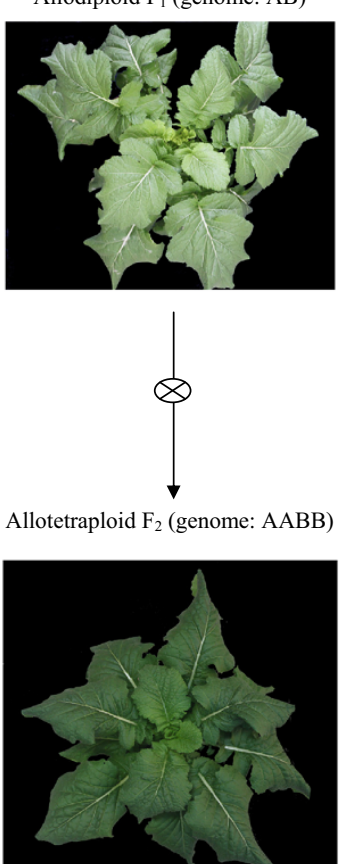

Figure 1 The layout of the experiment plants; the parents and their allodiploid and allotetraploid. 


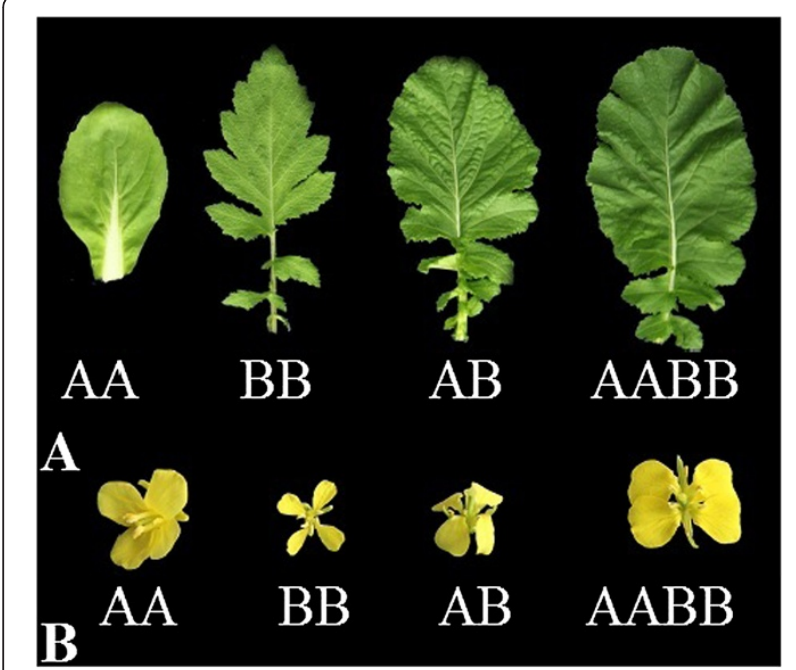

Figure 2 A. Leaves and B. flower of the parents and their allodiploid and allotetraploid. Allotetraploids had a high leaf length and flower size compared with their parents and the allodiploids.

including 60 categories according to the variation model between the parents and their allodiploids and allotetraploids (Additional file 1). Group A consisted of $12.22 \%$ and $12.08 \%$ of the monomorphic loci in $\mathrm{AB}$ and $\mathrm{AABB}$, respectively. In Group B, $11.94 \%$ of the polymorphic loci were specifically found in $A B$ and $A A B B$. Of the loci in Group C, $18.91 \%$ and $28.64 \%$ of the polymorphic loci were specifically found in $\mathrm{AB}$ and $\mathrm{AABB}$, respectively. Compared with the parents, the Group D loci displayed alterations in DNA methylation only in $56.94 \%$ and $48.10 \%$ of those found in $\mathrm{AB}$ and $\mathrm{AABB}$, respectively. There was a significant difference in the methylation patterns between $\mathrm{AB}$ and $\mathrm{AABB}$. With respect to the DNA methylation status, the ratios between the allodiploids, the allotetraploids, and their parents demonstrated significant differences
(Additional file 1). In addition, the CG methylation was high (24.50\%) in AB compared with AABB (Figure 3 and Additional file 1). Thus, the DNA methylation alteration in $A B$ was significantly higher compared with that in AABB. Moreover, the genetic study revealed that two types of the fragments can be used to estimate the genomic changes in the allodiploids and allotetraploids, which have a loss of fragments compared with the parents in addition to novel fragments. The percentages of genetic changes were $10.32 \%$ in $\mathrm{AB}$ and $21.41 \%$ in $\mathrm{AABB}$ (Figure 4 and Additional file 3). Thus, the percentage of genetic changes was significantly higher in AABB compared with $\mathrm{AB}$.

\section{High-throughput sequencing of small RNAs}

A small RNA library was prepared for the analysis of the parents and their allodiploids and allotetraploids. Sixty million reads were obtained from the small RNA sequencing of the four libraries (Additional file 4). A total of $41,810,504$ reads were obtained, and 41,664,822 of these reads were of high quality and corresponded to 28,426,693 unique sequence tags. The small RNA sizes ranged from 18 to $30 \mathrm{nt}$, which included the two prominent classes of 21-nt and 24-nt long small RNAs (Figure 5). The 21-nt class corresponded predominantly to miRNAs, whereas the 24-nt class corresponded to siRNAs [29]. The 24-nt long small RNAs were most abundant within the two parental and $A B$ libraries, whereas the 21-nt long small RNAs were most prevalent in the AABB library. Interestingly, the amount of miRNAs relative to the total small RNAs increased with increasing levels of polyploidy: the lowest percentages were $30.9 \%$ in $\mathrm{AA}, 29.34 \%$ in $\mathrm{BB}$, $31.44 \%$ in $\mathrm{AB}$, and $39.22 \%$ in $\mathrm{AABB}$. However, the amount of siRNAs relative to the total small RNA decreased with the increasing levels of polyploidy: the highest percentages were $23.93 \%$ in $\mathrm{AA}, 28.14 \%$ in $\mathrm{BB}, 26.90 \%$ in $\mathrm{AB}$, and

\section{$\square \mathrm{AA} \square \mathrm{BB} \square \mathrm{AB} \square \mathrm{AABB}$}

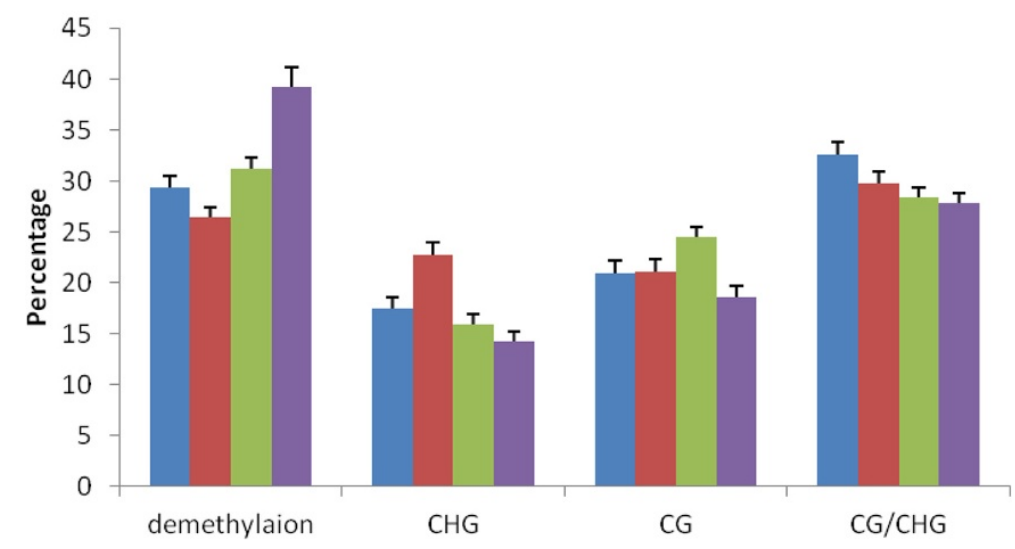

Figure 3 DNA methylation in the parents and their allodiploid and allotetraploid. 


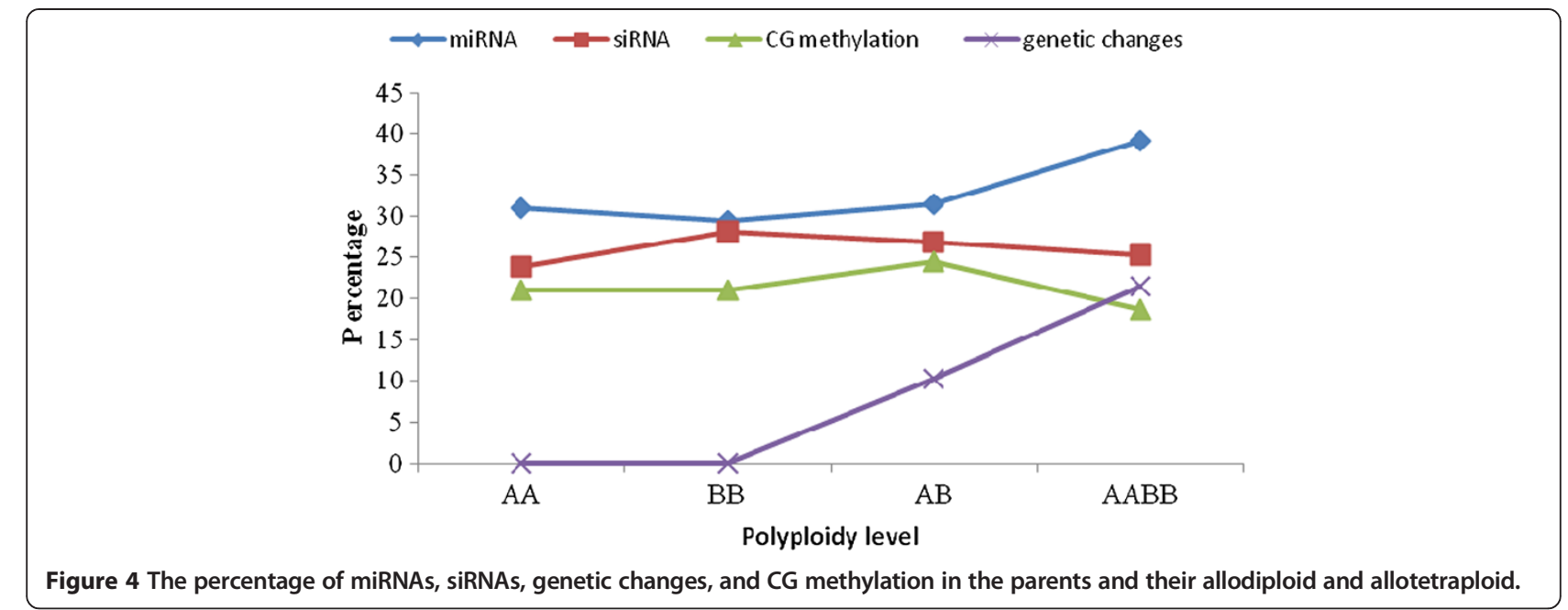

25.23\% in AABB (Figure 4). Moreover, the total small RNAs demonstrated a high interaction of $81.31 \%$ in $\mathrm{AA} / \mathrm{AB}$, and the unique small RNAs showed a high level of interaction of $15.34 \%$ in AA/AB (Additional file 4). The genome-matched small RNA tags were then clustered into several RNA categories (such as known mRNAs, repeatassociated RNAs, rRNAs, tRNAs, snRNAs, and snoRNAs) in the four libraries (Additional file 4). In addition, a high percentage of small RNAs were sorted as unann RNAs (44.81\% in AA, $44.23 \%$ in $\mathrm{BB}, 48.86 \%$ in $\mathrm{AB}$, and $53.55 \%$ in $A A B B)$. The repeat-associated sRNAs were matched based on LTR/Copia: 0, LTR/Copia: 1, LTR/Gypsy: 0, and LTR/Gypsy: 1 in both the unique tags and total tags. Unexpectedly, all four types of repeat-associated sRNAs accumulated in lower levels in AABB compared with $\mathrm{AB}$ (Additional file 5). Among the four types, the accumulation of 21-nt sRNAs was higher compared with that of 24-nt sRNAs.

\section{Known miRNAs}

To identify conserved miRNAs in the parents and their allodiploids and allotetraploids, small RNAs that were 18-23 nucleotides in length were searched using Blastn against miRBase version V17.0. The 22,954 and 22,716 unique sequences $(2,526,482$ and $2,176,470$ reads) that were found in the $\mathrm{AA}$ and $\mathrm{BB}$ libraries, respectively, were annotated as miRNA candidates. Totals of 24,143 and 23,061 unique sequences $(2,183,129$ and 2,524,964 reads) were found in the $\mathrm{AB}$ and $\mathrm{AABB}$ libraries, respectively (Additional file 4). The expression of known miRNAs in the four samples was demonstrated by plotting the $\log _{2}$-ratio (Additional file 6). These results showed 320 differentially expressed miRNAs: 133, 94, $132,143,134$, and 133 up-regulated miRNAs and 114, $66,103,110,95$, and 95 down-regulated miRNAs in $\mathrm{BB} /$ $\mathrm{AA}, \mathrm{AA} / \mathrm{AB}, \mathrm{AA} / \mathrm{AABB}, \mathrm{BB} / \mathrm{AB}, \mathrm{BB} / \mathrm{AABB}$, and $\mathrm{AB} /$ $A A B B$, respectively (Figure 6). These findings were

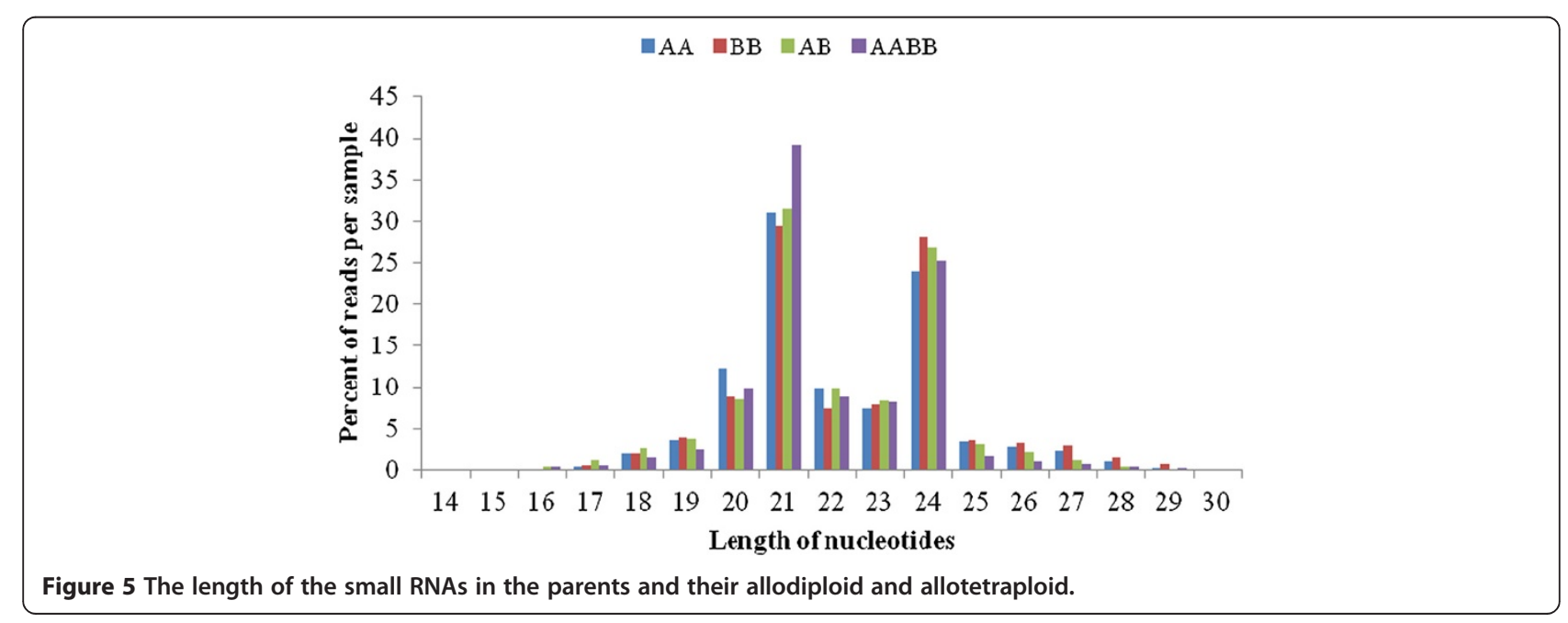




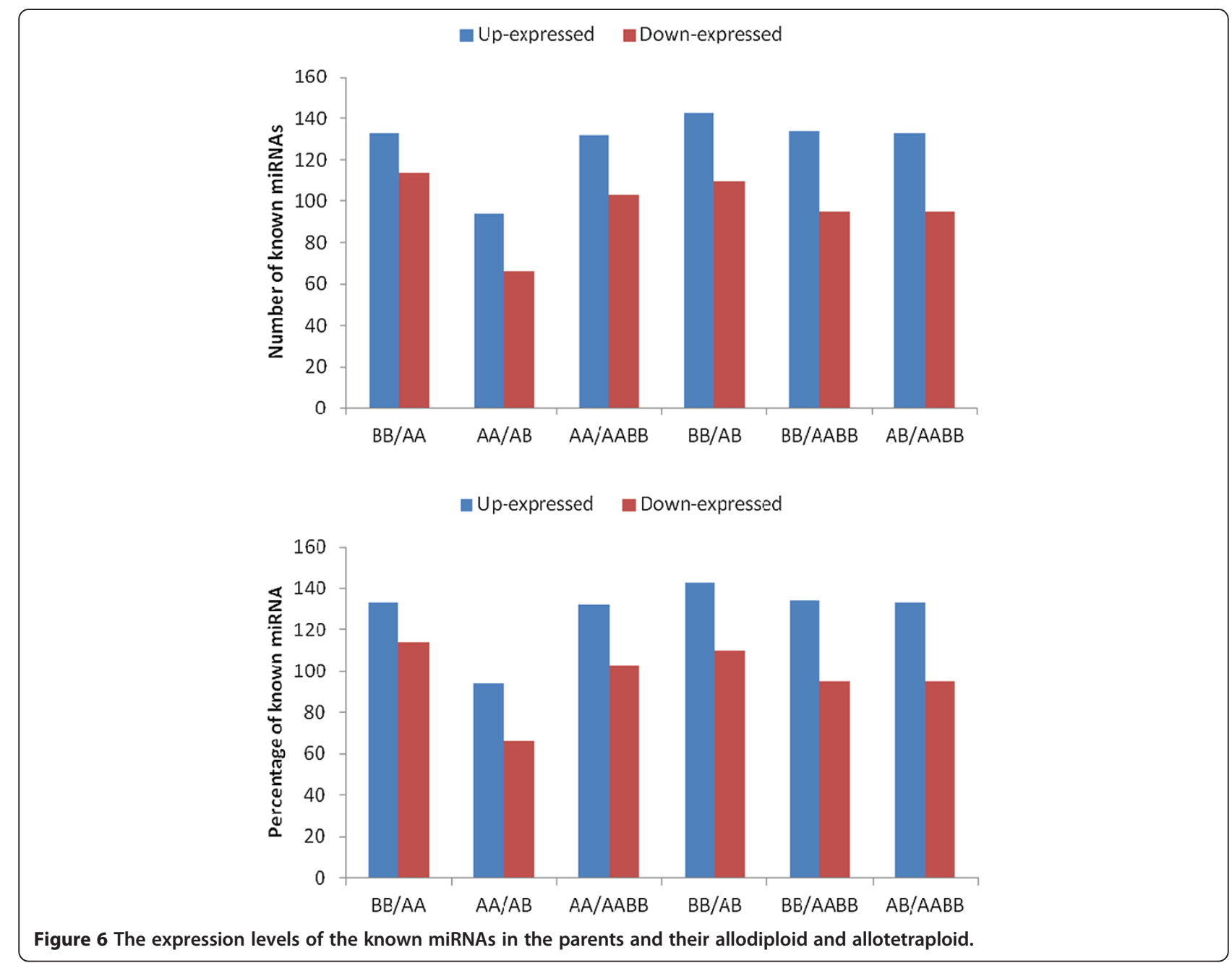

significantly different between the four libraries. In our study, further analysis identified totals of 1235, 2967, 1189 , and 2651 conserved miRNAs, which belong to 68 plants families, in the $\mathrm{AA}, \mathrm{BB}, \mathrm{AB}$, and $\mathrm{AABB}$ libraries, respectively (Additional file 7 ).

\section{Novel miRNAs}

The expression of novel miRNAs in the four samples was demonstrated by plotting the $\log _{2}$-ratio (Additional file 6). These results showed 52 novel miRNAs: 20,11 , $29,13,27$, and 24 up-regulated miRNAs and 16, 16, 7, 17,12 , and 7 down-regulated miRNAs in $\mathrm{BB} / \mathrm{AA}, \mathrm{AA} /$ $\mathrm{AB}, \mathrm{AA} / \mathrm{AABB}, \mathrm{BB} / \mathrm{AB}, \mathrm{BB} / \mathrm{AABB}$, and $\mathrm{AB} / \mathrm{AABB}$, respectively (Figure 7). These findings were significantly different between the four libraries.

\section{qRT-PCR analysis of miRNAs and their target genes}

Many miRNA targets play important roles in the regulation of their expression. The targets of the differentially expressed miRNAs were predicted to elucidate the relationship between functions and phenotypes. The prediction revealed a total of 641 known miRNAs and 3734 target genes: 158, 160, 159, and 164 known miRNAs and $895,972,895$, and 972 target genes in $\mathrm{AA}, \mathrm{BB}, \mathrm{AB}$, and $\mathrm{AABB}$, respectively (Additional file 6). Moreover, a total of 68 novel miRNAs and 225 target genes were detected: 11, 17, 14, and 56 novel miRNAs and 49, 53, 20, and 103 target genes in $\mathrm{AA}, \mathrm{BB}, \mathrm{AB}$, and $\mathrm{AABB}$, respectively (Additional file 6). Furthermore, we performed a quantitative analysis of nine miRNAs and 11 targets genes involved in different vegetative and reproductive functions. Moreover, the expression levels of the target genes (MYB65, CUC1, PHV, PHB, REV, NFYA2, APS1, APS4, and SULTR2:1) were higher in AB compared with AABB. However, the expression levels of SBP and AGO2 were higher in $\mathrm{AABB}$ compared with $\mathrm{AB}$ (Figure 8). Although the corresponding accumulated abundances of various miRNAs (miR159a, miR164a, miR165a, miR169i, miR395a, and miR403) were lower in $A B$ compared with $A A B B$, the levels of miR156a and miR157a were higher in AB compared with AABB (Figure 9). Moreover, only miR165a, miR166a, and miR395a were not inversely correlated with 


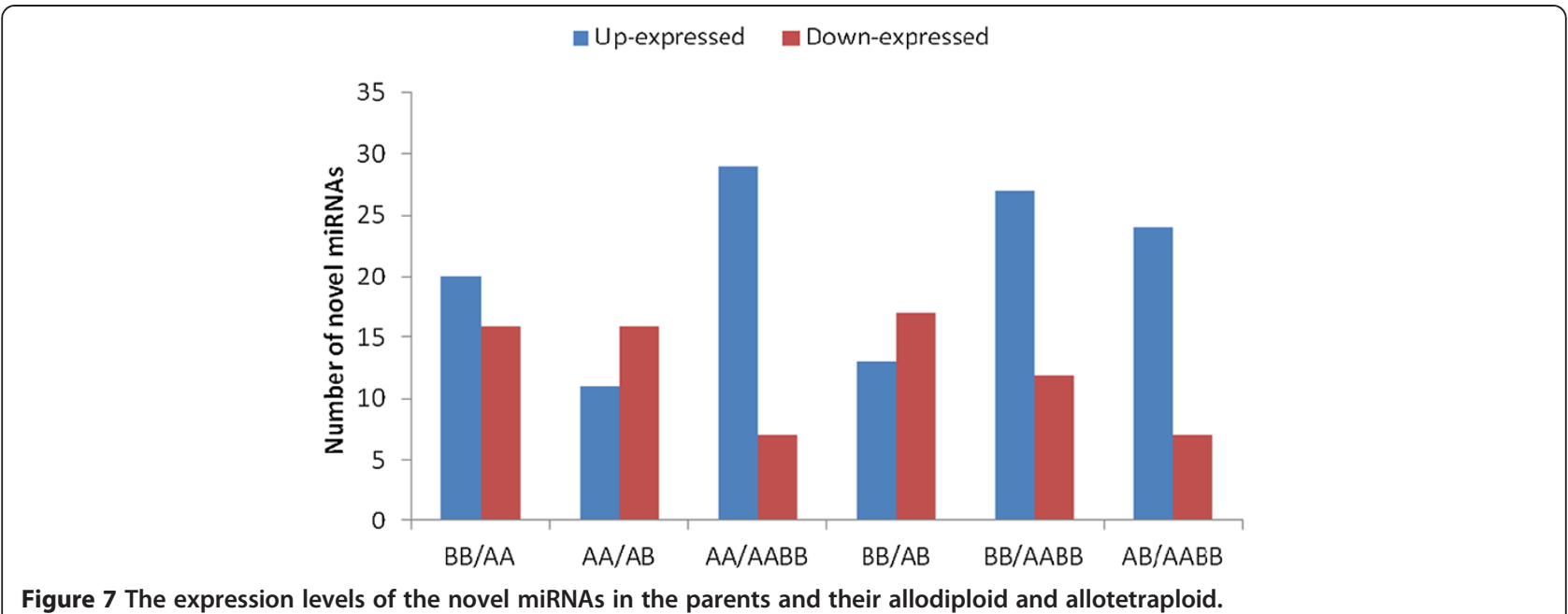

the targeted genes (REV, PHV, and APS1, SULTR2;1) in $A A B B$ and $A B$, respectively. Thus, the expression levels of the targets were negatively corrected with the abundance of significantly expressed miRNAs in this study.

\section{Discussion}

\section{Wide hybridisation and polyploidisation showed different} patterns of DNA methylation

In this study, the siRNAs and DNA methylation patterns were significantly different between the allodiploid (AB) and the allotetraploid (AABB). DNA methylation was mediated by 24-nt siRNAs, which are derived from repetitive DNA, transposons, and intergenic and genic regions $[24,30]$. Based on the length distribution of small RNAs, 24-nt siRNAs ranged from $26.90 \%$ (AB) to $25.23 \%$ (AABB) (Figure 5). The repeat-associated siRNAs were matched on the LTR (retrotransposons) and showed higher levels in AB compared with AABB (Additional file 5). The LTR can be reactivated by hybridisation, as has been demonstrated by several previous studies [31,32]. Thus, a hypothesis may be reached that the extent of retrotransponson activation varies depending on the wide hybridisation. Thus, the regulation of genomic dosage may display different patterns in $\mathrm{AB}$ and $\mathrm{AABB}$.

DNA methylation combined with the activation of a transposable element has been proposed as the stabilising mechanism underlying the epigenetic changes mediated by siRNAs during hybridisation and polyploidisation [13,33-35]. In response to genomic shock, the siRNAs maintain genomic stability in allopolyploids [13]. Thus, it can be speculated that siRNAs and DNA methylation are conducive to the maintenance of genome stability in AABB when faced with genomic shock in AB. Moreover, reduced siRNAs levels are largely associated with genes, and genes are associated with altered siRNAs levels and have correlations with changes in the DNA methylation and expression levels [36]. In the present study, the detected siRNAs and DNA methylation were low in the allotetraploids compared with the allodiploids. Thus, it has been proposed that siRNAs play a key role in maintaining the genomic stability of allotetraploids.

\section{Changes in siRNA and miRNA during wide hybridisation and polyploidisation}

siRNAs and miRNAs induce rapid and dynamic changes during the early stage of allopolyploid formation [13]. The most abundant small RNAs found in this study through high-throughput sequencing were identified as miRNAs and siRNAs, as assessed by the composition of miRNAs and siRNAs, which were often 21-nt and 24-nt in length, respectively [23]. The relative amount of small RNAs corresponding to miRNAs increased with an increase in the polyploidy level (Figure 4). Conversely, the relative amount of siRNAs corresponding to transposons decreased with an increase in the polyploidy level (Figure 4). Similarly, Cantu et al. [37] demonstrated that siRNAs corresponding to transposons are observed at lower levels in hexaploids compared with tetraploids. Moreover, the relative amount of epigenetic alterations decreased with an increase in the polyploidy level (Figure 4). Kenan-Eichler et al. [20] also reported that the percentage of siRNAs and epigenetic changes decreased and the miRNA levels increased in hexaploids. Thus, the miRNA expression increased with an increase in the polyploidy level, whereas the siRNA and epigenetic alteration levels decreased with an increase in the polyploidy level. Furthermore, this ploidy dependence was insensitive to genomic composition but was sensitive to dosage, such as that of $A B$ and $A A B B$, which featured the same two genomes (A and $\mathrm{B})$ at varying doses $(2 \times$ vs. $4 \times)$, resulting in their expression of divergent small RNAs profiles. 


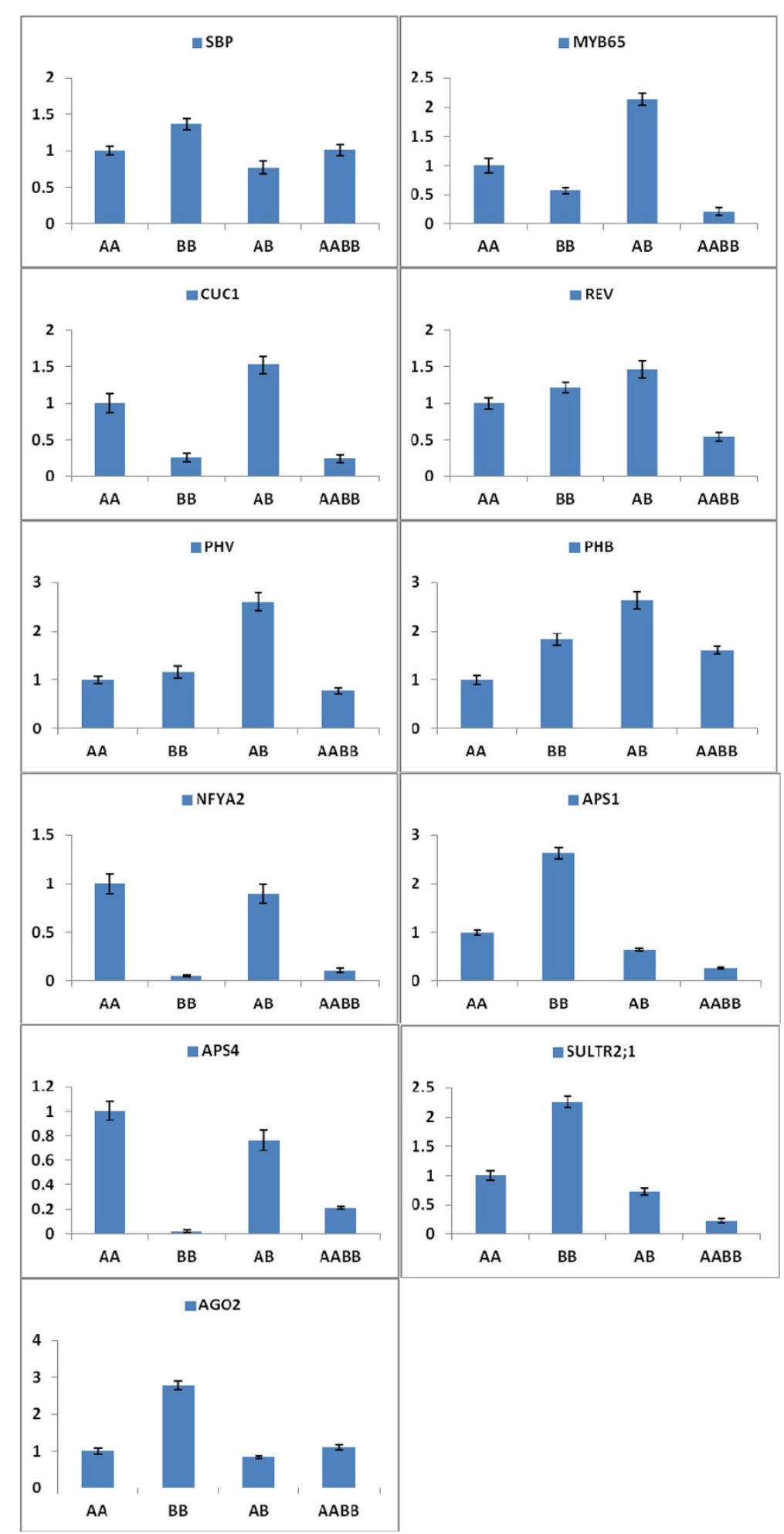

Figure 8 The expression of miRNA targets in the parents and their allodiploid and allotetraploid. 


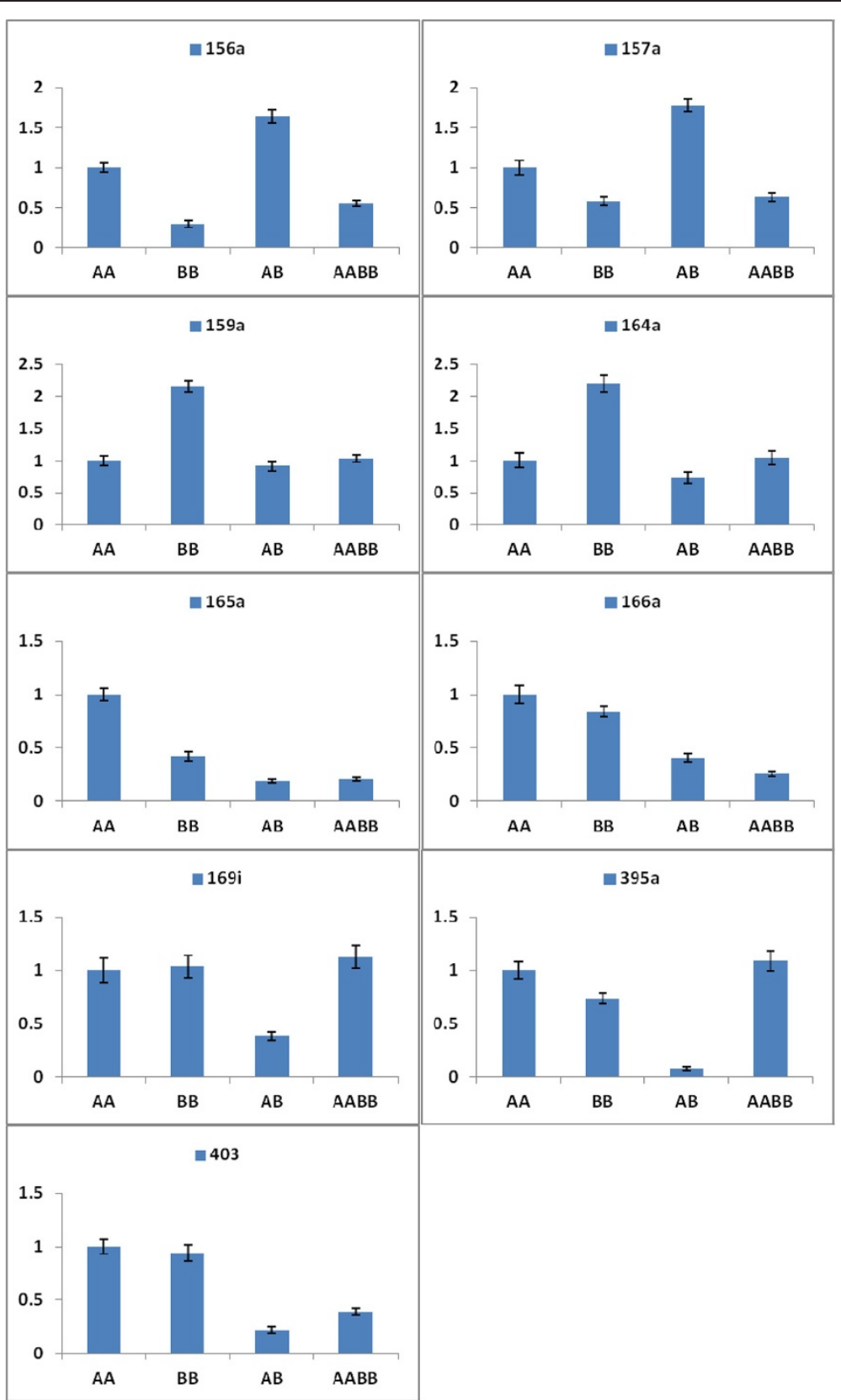

Figure 9 The expression levels of the miRNAs in the parents and their allodiploid and allotetraploid.

The expression of miRNAs and their target genes in the allodiploids and allotetraploids

miRNAs function as negative regulators of gene expression and are known to play markedly expanded roles in a variety of developmental processes affecting meristems, leaves, roots, and inflorescences [38]. The evolutionary conserved miR164, miR165, and miR166 regulate the development of leaves and contribute to the construction of leaf morphology. The overexpression of miR164, miR165 and miR166 reduce the levels of all CUC1, PHB, PHV, and REV genes and increase the development of SAM, which has effects on leaf development [39-42]. Similarly, the miR164a, miR165a, and miR166a levels were high and the CUC1, PHB, PHV, and REV levels were low in $\mathrm{AABB}$ compared with $\mathrm{AB}$ (Figures 9 and 8). In our previous study, $\mathrm{AB}$ and $\mathrm{AABB}$ presented significantly different phenotypes with respect to leaf length and width (Figure 2) [28].

In the vegetative to the reproductive phase, the targets of miR156, miR157, miR159 and miR169 participate in the activation of floral meristem identity genes. In Arabidopsis, as development proceeds, the decrease in the miR156/ 
miR157 levels and the increase in SPLs in the SAM result in the activation of floral meristem identity genes [43,44]. A high expression level of miR169 is known to have a positive effect on the timing and development of flowers downstream of NFYA2 [42,43,45,46]. Similarly, in our phenotypic study, flower development was high in $\mathrm{AABB}$ compared with $\mathrm{AB}$ (Figures 2, 9, and 8). Moreover, the overexpression of miR159 results in a delay in flowering, which is associated with a reduction in the levels of MYB [47-49]. In our findings, flower timing was delayed in AABB compared with $\mathrm{AB}$ (Figures 9 and 8 and Additional file 8).

A large number of miRNAs from diverse plants have been identified in the response to metal stress [50-52]. Recent studies have shown that miR395 is induced by sulphur starvation and regulates a low-affinity sulphate transporter (SULTR2;1) and three ATP sulphurylases (including APS1 and APS4) [50,53-55]. Furthermore, transgenic plants over-expressing miR395 accumulate more sulphate in the plant shoots, which suggests that miR395 is involved in the regulation of sulphate allocation by targeting APS genes and SULTR2;1 [55]. In our study, AABB plants were found to overexpress miR395 and presented low expression of its target genes (APS1, APS4, and SULTR2;1) compared with $\mathrm{AB}$ plants (Figures 9 and 8). These results showed that $\mathrm{AABB}$ plants exhibit tolerance to sulphate deficiency and heavy metal stress. Previous studies have shown that AGO2 mRNA is targeted by miR403 [54,56]. Moreover, the regulation of miRNAs and their targets may result in novel phenotypes in allopolyploids [13]. In addition, AABB plants presented a higher level of AGO2 compared with the AB plants (Figure 8). Taken together, the data suggest that miRNAs regulate gene expression and induce phenotype variation, such as heterosis, in allotetraploids.

This study explored the role of small RNAs in wide hybridisation and polyploidisation between $B$. rapa and $B$ nigra. The different miRNAs showed different expression levels in allodiploids and allotetraploids, which performed the phenotypic variation. However, some questions remain elusive, including how nonadditively expressed miRNAs and siRNAs affect growth and developmental traits, such as leaf shape, plant stature, biomass, flowering time, and fitness in allodiploids and allotetraploids, and whether and how siRNAs and DNA methylation play roles in this process. Therefore, further studies of the allodiploids and allotetraploids will be necessary.

\section{Conclusions}

This study explored the role of small RNAs in wide hybridisation and allopolyploidisation between Brassica rapa (genome: AA) and Brassica nigra (genome: $\mathrm{BB}$ ). When the A genome was crossed with the B genome,
siRNA levels increased and decreased relative to their parents; $B$. rapa and $B$. nigra, respectively, while the DNA methylation levels increased relative to their parents. When the genome AB was doubled, the siRNA and DNA methylation levels of the allotetraploid decreased compared with its allodiploid. When the A genome was crossed with the B genome, the miRNA levels increased relative to their parents. When the genome $A B$ was doubled, the miRNA levels of the allotetraploid increased compared with its allodiploid. This result showed that siRNAs, DNA methylation and miRNA play key roles in maintaining the genomic stability through the regulation of small RNA levels. Moreover, most miRNAs were highly overexpressed in the allotetraploid, which might be induced by the heterosis, such as miR159, miR169, and miR164, miR165, and miR166, which have a major role in flower and leaf development in the allotetraploid. Taken together, the findings of this study demonstrated that siRNAs and miRNAs maintain the genomic and phenotypic stability in the allotetraploid. Therefore, the present findings may provide new information for elucidating the effects of small RNAs on the formation of allopolyploidisation.

\section{Methods}

\section{Plant materials}

Wide hybridisation between $B$. rapa ( $q$, genome: AA) and $B$. nigra ( $\hat{O}$, genome: $\mathrm{BB})$ was performed to produce allodiploids $\left(\mathrm{F}_{1}\right.$, genome: $\left.\mathrm{AB}\right)$, and subsequently allotetraploids $\left(\mathrm{F}_{1}\right.$, genome: $\left.\mathrm{AABB}\right)$ were obtained by treating the allodiploids with $0.2 \%$ colchicine for $16 \mathrm{~h}$, as described in our pervious study reported by Ghani et al., [28]. After selfing of the allotetraploids, $\mathrm{F}_{2}$ allotetraploids were obtained (Additional file 8). All of the plants were grown in vermiculite mixed with $30 \%$ soil in a growth chamber under growth conditions of $22 / 18^{\circ} \mathrm{C}$ (day/night) and $16 \mathrm{~h}$ of illumination per day. The leaves from three plants of each type were collected 45 days after sowing in the vegetative stage for the analyses of the genetic and epigenetic alterations.

Sequence-related amplified polymorphism (SRAP) analysis was performed in the present study using twenty primer pairs (Additional file 2) according to a previously described method [57]. A modified version of the CTAB method was used to extract the genomic DNA [58]. To obtain reproducible and clear banding patterns, each amplification was repeated three times, and only bands showing consistent amplifications were scored.

\section{MSAP analysis}

Methylation-sensitive amplification polymorphism (MSAP) analysis was performed as described by Xiong et al. [59]. It contains double enzymes restriction (EcoRI, HpaII/ MspI), adapter ligation, pre-amplification and selective 
amplification. The primers used for selective amplification contained three selective nucleotides (Additional file 2). For the data analysis, values of 1 and 0 were used to represent the appearance of a fragment and the disappearance of a fragment in MSAP, respectively. Further, type $(1,1)$ represented the appearance of fragments in both the MspI and HpaII lanes, type $(1,0)$ represented the appearance of fragments only in the HpaII lanes, type $(0,1)$ represented the appearance of a fragment only in the MspI lane, and type $(0,0)$ represented the disappearance of fragments in both the MspI and HpaII lanes. To obtain reproducible and clear banding patterns, each amplification was repeated three times, and only bands showing consistent amplifications were scored.

\section{High-throughput sequencing of small RNAs}

To determine small RNA populations in B. rapa, B. nigra and their progenitors (both the allodiploid and the allotetraploid), small RNA libraries were generated from the leaves of the four genotypes, i.e., $B$. rapa (AA), B. nigra $(\mathrm{BB})$, the allodiploid (AB), and the allotetraploid (AABB). The total RNA was isolated using the Trizol reagent (Invitrogen, Carlsbad, CA, USA) according to the manufacturer's instructions and was sent to Beijing Genomics Institute (BGI) for sequencing. After treatment of the raw data, the clean sequences were subjected to further analyses as previously described [60]. The clean reads were analysed by length distribution and common sequences. The sequences were then matched to the genome of all of the plants to identify the repeat-associated sRNAs and to assess the expression of sRNAs. We identified known miRNAs using miRBase. To reveal the differential expression of miRNAs, the abundance of miRNAs in all of the libraries was normalised. The normalisation values were compared between the two libraries and were calculated in the form of fold-changes (fold-change $=\log 2$ (treatment/control). Moreover, the $p$-value was obtained using a previously described formula [61]. For the prediction of targets, the gene function, including the biological process, cellular component localisation, and molecular function of the genes were analysed. The small RNA sequence data are deposited in GEO with accession number GSE61872 (http://www.ncbi.nlm.nih.gov/geo).

\section{The expression of miRNAs and targeted genes using quantitative RT-PCR analysis}

Stem-loop qRT-PCR was used to quantify the miRNA shown to have a significantly different expression. The sequences of nine miRNAs were obtained from highthroughput sequencing, and primers were designed using the Primer software. Next, $2 \mu \mathrm{g}$ of the total RNA were converted into cDNA using complementary designed primers. Moreover, poly (A)-tailed qRT-PCR was used to quantify the expression of the target genes. Two $\mu \mathrm{g}$ of the total RNA were converted into cDNA using oligo (dT) primers. The specific forward and reverse primers were designed using GenScript. A total volume of $25 \mu \mathrm{l}$ containing $12.5 \mu \mathrm{l}$ of SYBR, $2.0 \mu \mathrm{l}$ of cDNA, $1.0 \mu \mathrm{l}$ of the forward primer, $1.0 \mu \mathrm{l}$ of the reverse primer, and $8.5 \mu$ of sterilised distilled water was amplified using ABI STEPONE Real-Time PCR. The cycling conditions were $95^{\circ} \mathrm{C}$ for $30 \mathrm{~s}$ followed by 40 cycles of $5 \mathrm{~s}$ at $95^{\circ} \mathrm{C}$ and $30 \mathrm{~s}$ at $60^{\circ} \mathrm{C}$. All of the reactions were performed in triplicate, and the U6 gene and $25 \mathrm{~s}$ rRNA served as references for the quantification of miRNAs and the target genes, respectively. The primers used in this study are shown in Additional file 2. The threshold cycle $(\mathrm{CT})$ values were obtained automatically using $\mathrm{ABI}$ STEPONE, and the fold changes for each gene were quantified as the relative quantity (RQ) values using $\mathrm{CT}$ $(2-\Delta \Delta \mathrm{Ct})$.

\section{Availability of supporting data}

The sequences data sets supporting the results of this article are available in GEO with accession number GSE61872 (http://www.ncbi.nlm.nih.gov/geo).

\section{Additional files}

\begin{abstract}
Additional file 1: Table S2. DNA methylation levels in the parents and their allodiploid and allotetraploid plants, as detected by the methylationsensitive amplified polymorphism (MSAP) assay. Table S3 A comparison of DNA methylation patterns based on the MSAP data between parents and their allodiploids and allotetraploids. Figure S3 DNA methylation in the parents $(A A$ and $B B$ ) and their allodiploid $(A B)$ and allotetraploid (AABB): (a) demethylation in all of the samples, (b) methylation in all of the samples, (c) demethylation in the offspring compared with the parents, and (d) methylation in the offspring compared with the parents.
\end{abstract}

Additional file 2: An Excel spreadsheet containing the primer of SRAP, the sequence of the MSAP adapters and primers used in this study, the primer sequence of the miRNA, and the primer sequence of the targeted gene.

Additional file 3: Table S1. Genetic alterations in the parents and their allodiploid and allotetraploid plants. Figure S2 Genetic alteration in the parents (AA and BB), the allodiploid (AB), and the allotetraploid (AABB): (a) novel fragments, (b) loss of fragments compared with the parents, and $(c, d)$ parents appear to fragment with offspring.

Additional file 4: Table S4. The summary of small RNA sequencing data. Table S5 The interaction between parents and their allodiploid and allotetraploid plants. Table S6 The distribution of the genome-mapped sequence reads in the small RNA libraries.

Additional file 5: An Excel spreadsheet containing the repeat $A A$, $B B, A B$, and $A A B B$.

Additional file 6: An Excel spreadsheet containing the known miRNA expression levels between the allodiploid and allotetraploid plants, the known miRNA expression levels between the parents and the allotetraploid, the known miRNA expression levels between the parent and the allotetraploid the known miRNA expression levels between the parents and the allodiploid, the known miRNA expression levels between the parents and the allodiploid, the known miRNA expression levels between the parents, and the novel miRNA expression levels between the allodiploid and the allotetraploid, the novel miRNA expression levels between the parents and the allotetraploid, the novel miRNA expression levels between the parents and the allotetraploid, the novel miRNA 
expression levels between the parents and the allodiploid, the novel miRNA expression levels between the parents and the allodiploid, the novel miRNA expression levels between the parents, and the known miRNA sequence $A A B B$, the known miRNA sequence $A B$, the known miRNA sequence $A A$, the known miRNA sequence $B B$, the novel miRNA sequence $A A B B$, the novel miRNA sequence $A B$, the novel miRNA sequence $A A$, the novel miRNA sequence $B B$, and the known miRNAs and prediction target genes in $A A$, the known miRNAs and prediction target genes in $B B$, the known miRNAs and prediction target genes in $A B$, the known miRNAs and prediction target genes in $A A B B$, and the novel miRNAs and prediction target genes in AA, the novel miRNAs and prediction target genes in $B B$, the novel miRNAs and prediction target genes in $A B$ and the novel miRNAs and prediction target genes in $A A B B$.

Additional file 7: An Excel spreadsheet containing the related crop family miRNAs.

Additional file 8: Figure S1. The number of days for the flowering of the parents and their allodiploid and allotetraploid plants.

\section{Competing interests}

The authors declare that they have no competing interest.

\section{Authors' contributions}

MAG and $J$ generated the SRAP, MSAP and sRNA data and interpreted the results. NY, MAR, LC and RL performed the qRT-PCR experiments. MAG drafted the manuscript. $L C, M A G, X Z$ and $J$ designed the research and did the statistical analyses. LC supervised the research. All authors read and approved the final manuscript.

\section{Acknowledgments}

This work was supported by the National Natural Science Foundation of China (No. 31272159), the Zhejiang Provincial Natural Science Foundation of China (No. LZ12C15001) and the Specialized Research Fund for the Doctoral Program of Higher Education (20110101110089). We thank Mr. Zhang Fenglan, the Beijing Vegetable Research Centre (BVRC), and the Beijing Academy of Agriculture and Forestry Science (BAAFS), Beijing, China for providing seeds (Brassica rapa)

Received: 5 May 2014 Accepted: 3 October 2014

Published online: 19 October 2014

\section{Reference}

1. Rieseberg LH, Willis JH: Plant speciation. Science 2007, 317:910-914.

2. Leitch AR, Leitch IJ: Genomic plasticity and the diversity of polyploid plants. Science 2008, 320:481-483.

3. Soltis PS, Soltis DE: The role of hybridization in plant speciation. Annu Rev Plant Biol 2009, 60:561-588

4. Wood TE, Takebayashi N, Barker MS, Mayrose I, Greenspoon PB, Rieseberg $\mathrm{LH}$ : The frequency of polyploid speciation in vascular plants. Proc Natl Acad Sci U S A 2009, 106:13875-13879.

5. Chen ZJ: Molecular mechanisms of polyploidy and hybrid vigor. Trends Plant Sci 2010, 15:57-71.

6. Chen S, Nelson MN, Chevre M, Jenczewski E, Li Z, Mason AS, Meng J, Plummer JA, Pradhan A, Siddique KHM, Snowdon RJ, Yan G, Zhou W, Cowling WA: Trigenomic bridges for Brassica improvement. Crit Rev Plant Sci 2011, 30(6):524-547.

7. Geng XX, Chen S, Astarini IA, Yan GJ, Tian E, Meng J, Li ZY, Ge XH, Nelson MN, Mason AS, Pradhan A, Zhou WJ, Cowling WA: Doubled haploids of novel trigenomic Brassica derived from various interspecific crosses. Plant Cell Tiss Org Cult 2013, 113(3):501-511.

8. Ehrendorfer F: Polyploidy and distribution: polyploidy Biological Relevance. In Basic Life Sciences Volume 13. 1st edition. Edited by Lewis WH. New York: Springer; 1980:45-60.

9. Wendel JF, Cronn RC: Polyploidy and the evolutionary history of cotton. Adv Agron 2003, 78:139-186.

10. Malek MA, Ismail MR, Rafii MY, Rahman M: Synthetic Brassica napus L.: development and studies on morphological characters, yield attributes, and yield. Sci W J 2012. doi:10.1100/2012/416901.
11. McClintock B: The significance of responses of the genome to challenge. Science 1984, 226:792-801.

12. Comai $L$ : The advantages and disadvantages of being polyploid. Nat Rev Genet 2005, 6:836-846.

13. Ha M, Lu J, Tian L, Ramachandran V, Kasschau KD, Chapman EJ, Carrington JC, Chen XM, Wang XJ, Chen ZJ: Small RNAs serve as a genetic buffer against genomic shock in Arabidopsis interspecific hybrids and allopolyploids. Proc Natl Acad Sci U S A 2009, 106:17835-17840.

14. Chapman EJ, Carrington JC: Specialization and evolution of endogenous small RNA pathways. Nat Rev Genet 2007, 8:884-896.

15. Molnar A, Melnyk C, Baulcombe DC: Silencing signals in plants: a long journey for small RNAs. Genome Biol 2011, 12:215.

16. Haag JR, Pikaard CS: Multisubunit RNA polymerases IV and V: purveyors of non-coding RNA for plant gene silencing. Nat Rev Mol Cell Biol 2011, 12:483-492.

17. Shivaprasad PV, Dunn RM, Santos BA, Bassett A, Baulcombe DC: Extraordinary transgressive phenotypes of hybrid tomato are influenced by epigenetics and small silencing RNAs. EMBO J 2012, 31:257-266.

18. Ng Danny W-K, Lu J, Chen ZJ: Big roles for small RNAs in polyploidy, hybrid vigor, and hybrid Incompatibility. Plant Biol 2012, 15:154-161.

19. Comai L, Tyagi AP, Winter K, Holmes-Davis R, Reynolds SH, Stevens Y, Byers B: Phenotypic instability and rapid gene silencing in newly formed Arabidopsis allotetraploids. Plant Cell 2000, 12:1551-1567.

20. Kenan-Eichler M, Leshkowitz D, Tal L, Noor E, Melamed-Bessudo C, Feldman M, Levy AA: Wheat hybridization and polyploidization results in deregulation of small RNAs. Genetics 2011, 188:263-272.

21. Chen ZJ: Genetic and epigenetic mechanisms for gene expression and phenotypic variation in plant polyploids. Annu Rev Plant Biol 2007 58:377-406.

22. Khraiwesh B, Arif MA, Seumel Gl, Ossowski S, Weigel D, Reski R, Frank W: Transcriptional control of gene expression by microRNAs. Cell 2010, 140:111-112.

23. Bartel DP: MicroRNAs: genomics, biogenesis, mechanism, and function. Cell 2004, 116:281-297.

24. Baulcombe D: RNA silencing in plants. Nature 2004, 431:356-363.

25. Axtell MJ: Evolution of microRNAs and their targets: are all microRNAs biologically relevant? Biochimicaet Biophysica Acta 2008, 1779:725-734.

26. Wang J, Tian L, Lee HS, Wei NE, Jiang H, Watson B, Madlung A, Osborn TC, Doerge RW, Comai L, Chen ZJ: Genome wide nonadditive gene regulation in Arabidopsis allotetraploids. Genetics 2006, 172:507-517.

27. Nikovics K, Blein T, Peaucelle A, Ishida T, Morin H, Aida M, Laufs P: The balance between the MIR164A and CUC2 genes controls leaf margin serration in Arabidopsis. Plant Cell 2006, 18:2929-2945.

28. Ghani MA, Qian S, Li J, Cao L, Rao L, Zou X, Chen L: Phenotypic and genetic variation occurred during in wide hybridisation and alloployploidisation between Brassica rapa and Brassica nigra. Sci Hort 2014, 176:22-31.

29. Ghildiyal M, Zamore PD: Small silencing RNAs: an expanding universe. Nat Rev Genet 2009, 10:94-108.

30. Kasschau KD, Fahlgren N, Chapman EJ, Sullivan CM, Cumbie JS, Givan SA, Carrington JC: Genome-wide profiling and analysis of Arabidopsis siRNAs. PLOS Biol 2007, 5(3):57-71.

31. Labrador $M$, Farre $M$, Utzet F, Fontdevila A: Interspecific hybridization increases transposition rates of Osvaldo. Mol Biol Evol 1999, 16:931-937

32. Liu B, Wendel JF: Retrotransposon activation followed by rapid repression in introgressed rice plants. Genome 2000, 43:874-880

33. Ha M, Ng DWK, Li WH, Chen ZJ: Coordinated histone modifications are associated with gene expression variation within and between species. Genome Res 2011, 21:590-598.

34. Adams KL, Wendel JF: Polyploidy and genome evolution in plants. Curr Opin Plant Biol 2005, 8:135-141.

35. Feldman M, Levy AA: Allopolyploidy a shaping force in the evolution of wheat genomes. Cytogenet Genome Res 2005, 109:250-258.

36. Groszmann M, Greaves IK, Albertyn ZI, Scofield GN, Peacock WJ, Dennis ES: Changes in 24-nt siRNA levels in Arabidopsis hybrids suggest an epigenetic contribution to hybrid vigor. Proc Natl Acad Sci U S A 2012, 108:2617-2622.

37. Cantu D, Vanzetti LS, Sumner A, Dubcovsky M, Matvienko M, Distelfeld f, Michelmore RW, Dubcovsky J: Small RNAs. DNA methylation and transposable elements in wheat. BMC Genomics 2010, 11:408. 
38. Chuck G, Candela H, Hake S: Big impacts by small RNAs in plant development. Curr Opin Plant Biol 2009, 12:81-86.

39. Kim J, Jung J, Reyes JL, Kim Y, Kim S, Kim JA, Lee M, Lee Y, Kim VN, Chua N, Park C: MicroRNA-directed cleavage of ATHB15 mRNA regulates vascular development in Arabidopsis inflorescence stems. Plant J 2005, 42:84-94.

40. Williams L, Grigg SP, Xie M, Christensen S, Fletcher JC: Regulation of Arabidopsis shoot apical meristem and lateral organ formation by microRNA miR166 gandits AtHD-ZIP target genes. Development 2005, 132:3657-3668.

41. Zhou G, Kubo M, Zhong R, Demura T, Ye Z: Overexpression of miR165 affects apical meristem formation, organ polarity establishment and vascular development in Arabidopsis. Plant Cell Physiol 2007, 48:391-404.

42. Li J, Wang Y, Zhang L, Liu B, Cao L, Qi Z, Chen L: Heritable variation and small RNAs in the progeny of chimeras of Brassica juncea and Brassica oleracea. J Exp Bot 2013, 64(16):4851-4862.

43. Wang JW, Czech B, Weigel D: miR156-regulated SPL transcriptionfactors define an endogenous flowering pathway in Arabidopsis thaliana. Cell 2009, 138:738-749.

44. Wu G, Park MY, Conway SR, Wang JW, Weigel D, Poethig RS: The sequential laction of miR156 and miR172 regulates developmental timing in Arabidopsis. Cell 2009, 138:750-759.

45. Gandikota M, Birkenbihl RP, Hohmann S, Cardon GH, Saedler H, Huijser P. The miRNA156/157 recognition element in the 3'UTR of the Arabidopsis SBP box gene SPL3 prevents early flowering by translational inhibition in seedlings. Plant J 2007, 49:683-693.

46. Allen RS, Li J, Stahle MI, Dubroue A, Gubler F, Millar AA: Genetic analysis reveals functional redundancy and them a jor target genes of the Arabidopsis miR159 family. Proc Natl Acad Sci U S A 2007, 104:16371-16376.

47. Achard P, Herr A, Baulcombe DC, Harberd NP: Modulation of floral development by agibberellin-regulated microRNA. Development 2004, 131:3357-3365.

48. Wilson RN, Heckman JW, Somerville CR: Gibberellin is required for flowering in Arabidopsis thaliana under short days. Plant Physiol 1992, 100:403-408.

49. Mallory AC, Bartel DP, Bartel B: MicroRNA-directed regulation of Arabidopsis AUXIN RESPONSE FACTOR17 is essential for proper development and modulates expression of early auxin response genes. Plant Cell 2005, 17:1360-1375.

50. Huang SQ, Xiang AL, Che LL, Chen S, Li H, Song JB, Yang ZM: A set of miRNAs from Brassica napus in response to sulfate-deficiency and cadmium stress. Plant Biotechnol J 2010, 8:887-899.

51. Xie FL, Huang SQ, Guo K, Zhu YY, Nie L, Yang ZM: Computational identification of novel microRNAs and targets in Brassica napus. FEBS Lett 2007, 581:1464-1473.

52. Zhou ZS, Zeng HQ, Liu ZP, Yang ZM: Genome-wide identification of Medicago truncatula microRNAs and their targets reveals their differential regulation by heavy metal. Plant Cell Environ 2012, 35:86-99.

53. Jones-Rhoades MW, Bartel DP: Computational identification of plant microRNAs and their targets, including a stress-induced miRNA. Mol Cell 2004, 14:787-799.

54. Allen E, Xie Z, Gustafson AM, Carrington JC: MicroRNA-directed phasing during trans-acting siRNA biogenesis in plants. Cell 2005, 121(2):207-221.

55. Liang G, Yang FX, Yu DQ: MicroRNA395 mediates regulation of sulfate accumulation and allocation in Arabidopsis thaliana. Plant J 2010, 62:1046-1057

56. Lobbes D, Rallapalli G, Schmidt DD, Martin C, Clarke J: SERRATE: a new player on the plant microRNA scene. EMBO Rep 2006, 7:1052-1058.

57. Li G, Quiros CF: Sequence-related Amplified Polymorphism (SRAP) a new marker system based on a simple, PCR reaction: its application to mapping, and gene tagging in Brassica. Theor App/ Genet 2001, 103:455-461.

58. Doyle JJ, Doyle JL: A rapid DNA isolation procedure for small quantities of fresh leaf tissue. Phytochem Bull 1987, 19:11-15.

59. Xiong LZ, Xu CG, Saghai Maroof MA, Zhang Q: Patterns of cytosine methylation in an elite rice hybrid and its parental lines, detected by a methylation-sensitive amplification polymorphism technique. Mol Gen Genet 1999, 261(3):439-446.
60. Sunkar R, Zhou X, Zheng Y, Zhang W, Zhu JK: Identification of novel and candidate miRNAs in rice by high throughput sequencing. BMC Plant Biol 2008, 8:25

61. Gao ZH, Shi T, Luo XY, Zhang Z, Zhuang WB, Wang L: High-throughput sequencing of small RNAs and analysis of differentially expressed microRNAs associated with pistil development in Japanese apricot BMC Genomics 2012, 13:371-384.

doi:10.1186/s12870-014-0272-9

Cite this article as: Ghani et al:: The role of small RNAs in wide hybridisation and allopolyploidisation between Brassica rapa and Brassica nigra. BMC Plant Biology 2014 14:272

\section{Submit your next manuscript to BioMed Central and take full advantage of:}

- Convenient online submission

- Thorough peer review

- No space constraints or color figure charges

- Immediate publication on acceptance

- Inclusion in PubMed, CAS, Scopus and Google Scholar

- Research which is freely available for redistribution

Submit your manuscript at www.biomedcentral.com/submit
C) Biomed Central 